\title{
SPECTRAL PROPERTIES OF ORDINARY DIFFERENTIAL OPERATORS GENERATED BY FIRST ORDER SYSTEMS
}

\author{
@ A.A. Shkalikov
}

avabanin@sfedu.ru

\section{УДК 517.977}

DOI: 10.33184/mnkuomsh1t-2021-10-06.32.

Мы рассматриваем спектральные свойства обыкновенных дифференциальных операторов, порожденных системами первого порядка.

Ключевые слова: спектральные свойства, дифференциальные операторы.

\section{Spectral properties of ordinary differential operators generated by first order systems}

We consider spectral properties of ordinary differential operators generated by first order systems.

Keywords: spectral properties, differential operators.

We consider operators generated by differential expressions of the form

$$
l(\mathbf{y})=\mathbf{B}(x) \frac{d \mathbf{y}}{d x}+\mathbf{A}(x) \mathbf{y}, \quad \mathbf{y}=\left\{y_{1}, y_{2}, \ldots, y_{n}\right\}, \quad x \in[a, b]
$$

and boundary conditions

$$
\mathbf{U}_{0} \mathbf{y}(a)+\mathbf{U}_{1} \mathbf{y}(b)=0 .
$$

Here $\mathbf{U}_{0}$ and $\mathbf{U}_{1}$ are $n \times n$ matrices, $\mathbf{B}=\operatorname{diag}\left\{b_{1}(x), b_{2}(x), \ldots, b_{n}(x)\right\}$, and it is assumed that $b_{j}^{-1}$ and the entries of the $n \times n$ matrix-function $\mathbf{A}$ are summable.

The results which we shall present in the talk depend essentially on conditions for the functions $b_{j}(x)$. The main results will be presented for the case when all the functions $b_{j}(x)$ are real and $b_{j}(x) \neq b_{k}(x) \neq 0$ for all $x \in[a, b]$ and $k \neq j$. This case corresponds to hyperbolic systems.

We modify the the concept of regularity (it was originated in the works of G.Birkhoff, J.Tamarkin, and R.Langer) and prove that the eigen and associated functions of a regular operator form an unconditional basis in $L_{2}(a, b)$. Some other properties of regular operators will also be discussed.

This investigation is supported by Russian Foundation of Fundamental Research, grant No 19-01-00240.

Shkalikov A.A., Lomonosov Moscow State University

Department of mathematics and mechanics 